\title{
JPEG 2000 SCALAR QUANTIZATION USING AN OPTIMALLY FREQUENCY LOCALIZED MODULATED LAPPED TRANSFORM
}

\author{
Peter C. Tay ${ }^{1}$ and Joseph P. Havlicek ${ }^{2}$ \\ ${ }^{1}$ Department of Electrical and Computer Engineering \\ University of Virginia, Charlottesville, VA 22904 \\ ${ }^{2}$ School of Electrical and Computer Engineering \\ University of Oklahoma, Norman, OK 73019
}

\begin{abstract}
JPEG 2000 is a lossy integer-to-integer transform-based compression method that first quantizes the separable 2-D wavelet transform coefficients, then entropy codes them. The image is restored by performing an inverse wavelet transform on the dequantized and decoded coefficients. In this paper we consider closely related coding strategies using modulated lapped transforms rather than wavelet transforms, where performance is studied as a function of time (space) localization, frequency localization, and joint localization. In terms of reconstruction error at a given coding gain (quantization step size), we find that the modulated lapped transform admitting the best frequency localization offers superior performance relative to both other lapped transforms and orthogonal wavelet transforms.
\end{abstract}

\section{INTRODUCTION}

In the maximally decimated system shown in Fig. 1, there are $L$ analysis and $L$ synthesis filters. The decimation and interpolation factors between the analysis and synthesis filters is also given by $L$. If the output of this system is equal to the input up to a translation, then the system is considered a maximally decimated perfect reconstruction system. It is well-known that any such maximally decimated perfect reconstruction system can be realized by a modulated lapped transform (MLT) as described as in [1].

A tutorial on design considerations for constructing maximally decimated perfect reconstruction filter banks is given in [2], while a more thorough treatment appears in [3]. The goal of this paper is similar to the one in [4], where the filter bank is designed so that the coding gain is maximized. We have yet to investigate the improvements to the coding gain measure as prescribed in [4]. Rather, we will show that when the discrete wavelet transform (DWT) is replaced by an MLT filter bank, choosing the filter bank with optimal

This work was supported in part by the U.S. Army Research Laboratory and the U.S. Army Research Office under grant W911NF-04-1-0221. frequency localization delivers reconstruction performance with a resiliency against JPEG 2000 integer-to-integer quantization errors that is superior both to other MLT's and to the DWT.

The discrete-discrete measures we use to quantify the time and/or frequency localization of an FIR filter are given in [5]. These measures are invariant under time translation, frequency translation, phase shifts, and (by duality) modulations. They will be used to quantify the time (space), frequency, and joint localization of the filters which constitute an MLT.

In [6], Adams describes a method used in the lossy JPEG 2000 compression standard that quantizes the coefficients of a multi-level integer-to-integer DWT. The quantized coefficients are entropy coded, then the decoded, dequantized coefficients are transformed using an inverse DWT (IDWT) to reconstruct the image. Though the DWT and IDWT generally constitute a perfect reconstruction system, reconstruction errors still occur due to the quantization and subsequent dequantization steps.

The quantizer that is used in the JPEG 2000 compression standard is given by

$$
Q[n, m]=\operatorname{sgn}\{w[m, n]\}\left\lfloor\frac{|w[m, n]|}{\lambda}\right\rfloor,
$$

where $\mathbf{w}=\operatorname{DWT}\{\mathbf{I}\}$ for some image $\mathbf{I}$ and $\lfloor x\rfloor$ refers to the greatest integer less than or equal to $x$. The quantization step size is $\lambda$ and is equal to $2^{p}$ for some positive integer $p$. If the transform coefficients are coded as $2^{q}$ bit integers where $q$ is a positive integer greater than $p$, then the quantization in equation (1) equates to using the $(q-p)$ most significant bits to code the transform coefficients.

To pseudo-invert the quantization, the following dequantization step is performed:

$$
D[n, m]=\lambda(Q[n, m]+T \operatorname{sgn}\{Q[n, m]\}),
$$

where $T$ represents the bias with default value 0.5 .

JPEG 2000 performs the reconstruction via IDWT on the dequantized coefficients. In this paper, we replace 


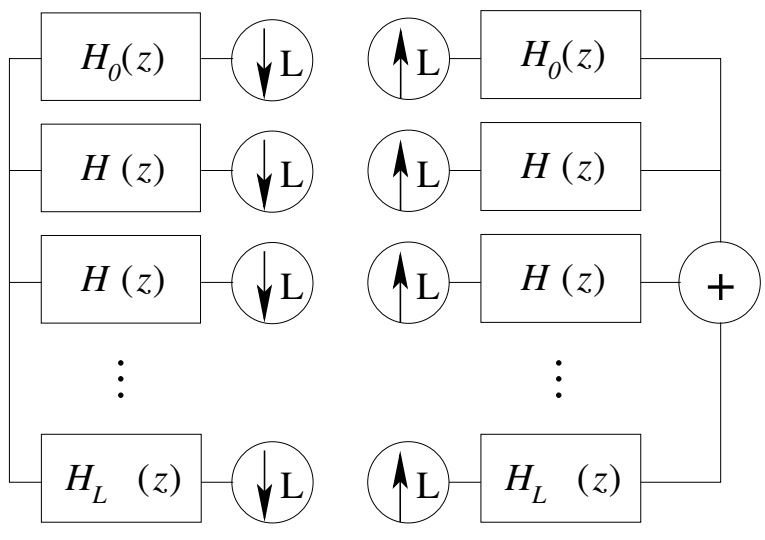

Fig. 1. Maximally decimated filter bank.

the reversible DWT system with a reversible MLT system. In Section 4, we provide experimental evidence that the MLT which consists of the best frequency localized filters provides superior reconstruction performance against other MLT's and even against the usual DWT system.

\section{MODULATED LAPPED TRANSFORM}

Malvar in [1] describes a method to construct multi-channel maximally decimated perfect reconstruction filter banks using discrete cosine transforms. The approach eliminates discontinuities which may occur in processing blocks of data by lapping adjacent blocks. In addition, the synthesis filters constitute an orthogonal set. Thus, this transform is referred to as the lapped orthogonal transform (LOT). The MLT is a specific class of LOT where the length of each analysis and synthesis filter is twice the number of channels. The synthesis filters are obtained from the corresponding analysis filters by time reversal. The analysis filters are constructed by modulating a low pass prototype filter, i.e., a window. The analysis filters of the 1-D $L$-channel MLT are defined as

$$
h_{k}[n]=\sqrt{\frac{2}{L}} w[n] m_{k}[n]
$$

where

$$
m_{k}[n]=\cos \left[\left(n+\frac{2 L+1}{2}\right)\left(k+\frac{1}{2}\right) \frac{\pi}{2 L}\right],
$$

$k=0,1,2, \ldots, L-1$ and $n=0,1,2, \ldots, 2 L-1$. The MLT can be extended to a complex MLT (CMLT) when

$$
m_{k}[n]=e^{j\left[\left(n+\frac{2 L+1}{2}\right)\left(k+\frac{1}{2}\right) \frac{\pi}{2 L}\right]},
$$

in which case equation (3) then defines a CMLT.

The system in Fig. 1 constitutes a perfect reconstruction system when the window $w[n] \in \mathbb{R}$ in equation (3) satisfies the following two conditions:

$$
\begin{aligned}
& \text { 1. } w^{2}[n]+w^{2}[n+L]=1 \text {, and } \\
& \text { 2. } w[n]=w[2 L-1-n] \text {. }
\end{aligned}
$$

The filter bank output is then equal to the input up to an integer translation. Cascading each synthesis filter with its corresponding analysis filter results in a linear phase impulse response [1], which ensures that the sum of the outputs of each channel is equal to an integer delayed version of the input signal. Separable multidimensional MLT's can be constructed from the 1-D transform in a straightforward way by taking separable products of the 1-D channel transfer functions.

\section{DEFINING LOCALIZATION}

We briefly review the translation and modulation invariant time and frequency localization measures given in [5]. Consideration is restricted to length- $N$ FIR filters with unit $\ell_{[0, N-1]}^{2}$-norm. If a finite length non-zero sequence is not unit $\ell_{[0, N-1]}^{2}$-norm, then the localization of that sequence is taken to be the measure applied to the unity-norm normalized sequence. Let $h:[0, N-1] \longrightarrow \mathbb{C}$ be a finite length sequence such that

$$
\sum_{n=0}^{N-1}|h[n]|^{2}=1=\frac{1}{N} \sum_{k=0}^{N-1}|H[k]|^{2},
$$

where $H[k]$ is the $N$-point DFT of $h[n]$. We can consider $|h[n]|^{2}$ to be a probability density function in discrete time and $\frac{1}{N}|H[k]|^{2}$ to be a probability density function in discrete frequency. These densities describe, respectively, how the energy of the signal is distributed in time and in frequency. Consider $n$ and $k$ to be a random variables in $[0, N-1]$. The variance in time of $\mathbf{h}$ is defined by the second central moment

$$
\sigma_{n, \mathbf{h}}^{2}=\sum_{n=0}^{N-1}(n-\mu)^{2}|h[n]|^{2},
$$

where $\mu$ is the expected value of $n$, also known as the mean or first moment, defined by

$$
\mu=\sum_{n=0}^{N-1} n|h[n]|^{2} .
$$

The variance in frequency of $h[n]$ will be computed from $H[k]$ according to

$$
\sigma_{\omega, \mathbf{h}}^{2}=\frac{1}{N} \sum_{k=0}^{N-1}(k-\nu)^{2}|H[k]|^{2},
$$

where the mean in discrete frequency is

$$
\nu=\frac{1}{N} \sum_{k=0}^{N-1} k|H[k]|^{2} .
$$


If we exclude filters that have a zero time or frequency variance such as the Kronecker Delta, the constant, and certain sinusoidal sequences, then a relation analogous to the Heisenberg-Weyl Uncertainty Principle as defined by Gabor in [7] holds, i.e.,

$$
\gamma_{N, \mathbf{h}}^{2} \equiv \sigma_{n, \mathbf{h}}^{2} \sigma_{\omega, \mathbf{h}}^{2} \geq C>0 .
$$

The localization measures defined in equations (7), (9), and (11) are not shift invariant in either the time or frequency domains. This means that the statement $f[n]=$ $g\left[\left(n-m_{t}\right) \bmod N\right]$ for some $m_{t} \in \mathbb{Z}$ does not necessarily imply that $\sigma_{n, \mathbf{g}}^{2}=\sigma_{n, \mathbf{f}}^{2}$. Likewise, the statement $F[k]=G\left[\left(k-m_{f}\right) \bmod N\right]$ for some $m_{f} \in \mathbb{Z}$ also does not necessarily imply that $\sigma_{\omega, \mathrm{g}}^{2}=\sigma_{\omega, \mathrm{f}}^{2}$. The shifting for a finite length sequence is defined to be circular shifting (also known as rotation): the values to be shifted in are taken from the periodic extension of the signal.

To make the measures (7), (9), and (11) invariant under translations and modulations, the localization measure of a sequence is defined by considering the sequence as an element of an equivalence class. Let $\mathbb{S}=$ $\{\mathbf{h} \mid \mathbf{h}$ is a length $N$ sequence $\}$.

Definition 1 Let $\mathbf{f}, \mathbf{g} \in \mathbb{S}$. Define a relation between these two sequences as $\mathbf{f} \sim \mathbf{g}$ if $\exists p, q, r \in \mathbb{Z}$ such that

$$
g[n]=e^{j \frac{2 \pi}{N}(q n+r)} f\left[(n-p)_{N}\right] .
$$

It is an easy exercise to show that the relation $\sim$ in definition 1 is reflexive, symmetric, and transitive. Thus, it defines an equivalence relation on $\mathbb{S}$. For a sequence $\mathbf{f} \in \mathbb{S}$, the equivalence class $[\mathbf{f}]$ is defined by

$$
[\mathbf{f}]=\{\mathbf{g} \in \mathbb{S} \mid \mathbf{g} \sim \mathbf{f}\}
$$

Theorem 1 Let $\mathbf{f}$ and $\mathbf{g}$ be two length $N$ sequences. Then $\mathbf{f} \sim \mathbf{g}$ if and only if $\mathbf{F} \sim \mathbf{G}$.

Theorem 1 establishes that, as an operator, the DFT preserves equivalence classes under the equivalence relation given in definition 1: every member of $[\mathbf{f}]$ has a DFT that is a member of $[\mathbf{F}]$ and every member of $[\mathbf{F}]$ has an IDFT that is a member of $[\mathbf{f}]$. Therefore, the time localization of $\mathbf{f}$ is quantified by the minimum time variance achieved by any $\mathbf{g} \in[\mathbf{f}]$,

$$
\sigma_{n,[\mathbf{f}]}^{2}=\min _{\mathbf{g} \in[\mathbf{f}]}\left\{\sigma_{n, \mathbf{g}}^{2}\right\} .
$$

Likewise, the frequency localization of $\mathbf{F}$ is quantified by the minimum frequency variance achieved by any $\mathbf{H} \in[\mathbf{F}]$. According to Theorem 1, this is precisely equivalent to quantifying the frequency localization of $\mathbf{f}$ by the minimum frequency variance achieved by any $\mathbf{h} \in[\mathbf{f}]$,

$$
\sigma_{\omega,[\mathbf{f}]}^{2}=\min _{\mathbf{h} \in[\mathbf{f}]}\left\{\sigma_{\omega, \mathbf{h}}^{2}\right\} .
$$

\begin{tabular}{||c|c|c|c|c||}
\cline { 3 - 5 } \multicolumn{2}{c|}{} & $\sigma_{\omega,\left[\widehat{\mathbf{h}}_{0}\right]}^{2}$ & $\gamma_{N,\left[\widehat{\mathbf{h}}_{0}\right]}^{2}$ & $\sigma_{n,\left[\widehat{\mathbf{h}}_{0}\right]}^{2}$ \\
\hline \multirow{2}{*}{$\mathrm{L}$} & Best Freq. & 0.2500 & 0.8176 & 3.2702 \\
\cline { 2 - 5 } $\mathrm{T}$ & Best Conjoint & 0.2642 & 0.7725 & 2.9244 \\
\cline { 2 - 5 } & Best Time & 3.5037 & 7.8834 & 2.2500 \\
\hline
\end{tabular}

Table 1. Time, frequency, and joint localizations of the 5channel MLTs having optimal localization of each type.

The conjoint localization of $\mathbf{f}$ simultaneously in time and frequency is quantified by the measure $\gamma_{N, \mathbf{f}}^{2}$ defined on $[\mathbf{f}]$ according to

$$
\gamma_{N, \mathbf{f}}^{2}=\sigma_{n,[\mathbf{f}]}^{2} \sigma_{\omega,[\mathbf{f}]}^{2} .
$$

\section{EXPERIMENTAL RESULTS}

Since the filters in the analysis filter bank of a CMLT are related by modulation, they all belong to the same equivalence class and thus have identical time and frequency localizations. We define localization of the MLT as that of the complex extension of the filter $h_{0}[n]$. Explicitly, let $h_{l}[n]$ be a filter in an MLT filter bank. Then

$$
\begin{aligned}
\sigma_{n,\left[\mathbf{h}_{l}\right]}^{2} & =\sigma_{n,\left[\widehat{\mathbf{h}}_{0}\right]}^{2}, \\
\sigma_{\omega,\left[\mathbf{h}_{l}\right]}^{2} & =\sigma_{\omega,\left[\widehat{\mathbf{h}}_{0}\right]}^{2}, \\
\gamma_{N,\left[\mathbf{h}_{l}\right]}^{2} & =\gamma_{N,\left[\widehat{\mathbf{h}}_{0}\right]}^{2},
\end{aligned}
$$

where $\widehat{\mathbf{h}}_{0}$ is the complex extension of $\mathbf{h}_{0}$.

By varying the FIR coefficients of the MLT window, an exhaustive search was performed to find the fivechannel MLTs admitting the best time localization, best frequency localization, and best joint localization as defined by (17), (18), and (19), resp. The results of this search are listed in Table 1, where it is evident that the reciprocal relationship between time and frequency localization as expressed by the Heisenberg-Weyl inequality is maintained.

Using the $256 \times 256$ pixel gray scale image lena with eight bits per pixel, we performed the JPEG 2000 quantization scheme with various step sizes $\lambda$. After dequantization (using the default bias) and upsampling, the image was reconstructed by summing the outputs of the synthesis filters. We compared performance of the MLTs indicated in Table 1 and the DWT based on mean reconstruction error defined by $M E=\sqrt{\sum|\mathbf{I}-\mathbf{J}|^{2}} / 256$, where $\mathbf{I}$ and $\mathbf{J}$ are the original and reconstructed images, respectively.

Fig. 2 show the original and reconstructed lena images using various MLTs with $\lambda=32$ and $T=0.5$. Fig. 3 gives the mean error associated with each MLT for a wide range of $\lambda$ 's with $T=0.5$. The results of this experiment using the JPEG 2000 default 5/3 DWT is also depicted in Fig. 3. 


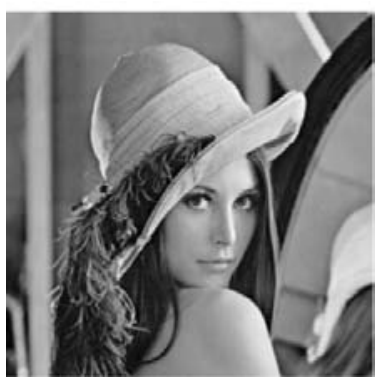

(a) Original image

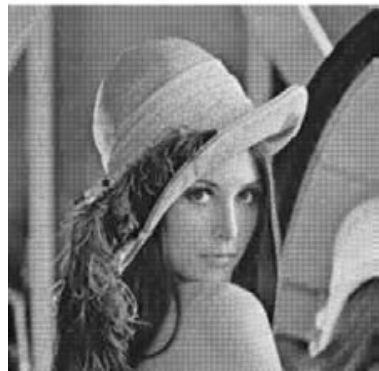

(c) Best conjointly localized MLT.

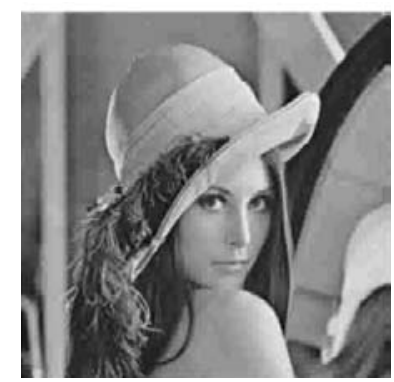

(b) Best frequency localized MLT.

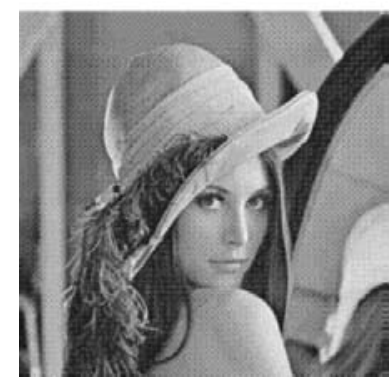

(d) Best time localized MLT.
Fig. 2. Original image and reconstructions using various MLTs with $\lambda=32$ and $T=0.5$.

While the DWT performs reasonably well, it is unable to outperform the best frequency localized MLT, especially at large $\lambda$. Both Fig. 2 and 3 suggest that the better frequency localized the MLT, the more resilient the system is to JPEG 2000 quantization and dequantization.

\section{CONCLUSION}

In this paper, we replaced the DWT used in the JPEG 2000 compression standard with several MLTs having optimal time, frequency, or conjoint localization. The localization measures used were based on variances in time and in frequency. These measures are defined on novel equivalence classes of FIR filters to obtain translation and modulation invariance. We performed the JPEG 2000 quantization and dequantization algorithms using the default $5 / 3$ DWT and all three optimally localized MLTs. We found that the MLT admitting optimal frequency localization consistently outperformed the other transforms in terms of mean reconstruction error over a wide range of quantization step sizes. While this experiment is certainly limited in scope, it strongly suggests that frequency localization is a powerful predictor of fidelity and performance in JPEG 2000 applications. Important future work will include comprehensive testing of this hypothesis on a large, diverse image database and bit rate comparison after entropy encoding the transform coefficients.

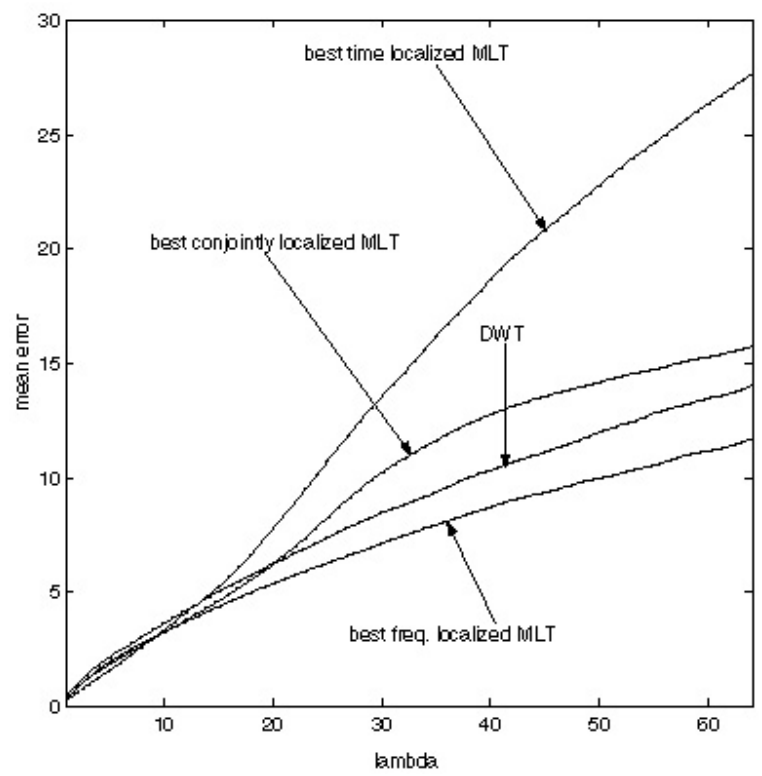

Fig. 3. $M E$ for DWT and several MLTs as a function of $\lambda$.

\section{REFERENCES}

[1] H. S. Malvar, "Lapped transforms for efficient transform/subband coding," IEEE Trans. Acoustic Speech and Signal Processing, vol. 38, no. 6, pp. 969-978, June 1990.

[2] T. P. Barnwell III and M.J.T. Smith, "Filter banks for analysis-reconstruction systems: a tutorial," in Proc. IEEE Int'l. Symp. Circuits, Syst., New Orleans, LA, May 1-3 1990, pp. 1999-2003.

[3] P.P. Vaidyanathan, "Optimal design of linear-phase FIR digital filters with very flat passbands and equiripple stopbands," IEEE Trans. Circ. Syst., vol. CAS-32, no. 9, pp. 904-917, Sept. 1985.

[4] A. Kirac and P.P. Vaidyanathan, "Theory and design of optimum FIR compaction filters," IEEE Trans. Signal Proc, vol. 46, no. 4, pp. 903-919, April 1998.

[5] P. C. Tay, J. P. Havlicek, and V. DeBrunner, "A novel translation and modulation invariant discrete-discrete uncertainty measure," in Proc. IEEE Int'l. Conf. Speech and Signal Proc., Orlando, FL, May 13-7 2002, pp. 1461-1464.

[6] M. D. Adams, "The JPEG-2000 still image compression standard," ISO/IEC JTC 1/SC 29/WG 1 N 2412, Dec. 2002.

[7] D. Gabor, "Theory of communication," J. Inst. Elect. Eng. London, vol. 93, no. III, pp. 429-457, 1946. 